\title{
Recurrent Catamenial Pneumothorax Caused by Diaphragmatic Fenestration
}

\author{
Burçin Çelik MD, Selçuk Gürz MD, Mehmet Gökhan Pirzirenli MD, and Mehmet Kefeli MD
}

\section{Introduction}

Catamenial pneumothorax, which is a rare cause of spontaneous pneumothorax, is defined as a pneumothorax that starts at the onset of or within the first 72 hours of the menstruation period in women. ${ }^{1}$ Catamenial pneumothorax was first described by Maurer et al in 1958. ${ }^{2}$ The reported incidence of catamenial pneumothorax among the spontaneous pneumothoraces is $3-6 \%$, but the actual incidence is $25-30 \%$, because catamenial pneumothorax is under-diagnosed., ${ }^{1,3}$ Although catamenial pneumothorax is clinically similar to the other primary spontaneous pneumothoraces, it is distinguished by being observed in women of reproductive age and by recurrence in menstrual periods.

The pathophysiology of catamenial pneumothorax is uncertain. Thoracic endometriosis or subpleural endometrial implants seem to be the most realistic explanation. If thoracic endometriosis is not detected, diaphragmatic fenestrations should be considered. In that situation, air enters the peritoneal cavity through the genital tract during menstruation, then, passes into the right hemithorax through the fenestrations on that side of the diaphragm, and causes pneumothorax..$^{4,5}$

We report a patient with catamenial pneumothorax who underwent repeated thoracotomy for recurrent pneumothorax, which revealed diaphragmatic fenestration during the operation.

\footnotetext{
The authors are affiliated with the Department of Thoracic Surgery, with the exception of Dr Kefeli, who is affiliated with the Department of Pathology, Ondokuz Mayis University Medical School, Samsun, Turkey.

Dr Çelik presented a version of this paper at the 16th Annual Meeting of the Turkish Thoracic Society, held April 3-7, 2013, in Antalya, Turkey.

The authors have disclosed no conflicts of interest.

Correspondence: Burçin Çelik MD, Department of Thoracic Surgery, Ondokuz Mayis University Medical School, Kurupelit, 55139, Samsun, Turkey. E-mail: cburcin@hotmail.com.
}

DOI: $10.4187 /$ respcare.02496

\section{Case Summary}

A 33-year-old woman presented with the complaint of chest pain and a history of recurrent pneumothoraces. Chest $\mathrm{x}$-ray revealed pneumothorax on the right side, and she was referred to our clinic for further evaluation and treatment (Fig. 1). According to her history, the first pneumothorax occurred on the right side, 5 years prior, and she was only observed, without placement of chest tube. Two months before we saw her, she had another pneumothorax, in the right hemithorax. A chest tube was placed at another hospital, and she was followed for 15 days with chest tube, due to prolonged air leak. Then she underwent partial decortication and apical wedge resection via right axillary thoracotomy. During the operation, no bullae, blebs, or endometrial tissue was observed, and she was discharged with full recovery. Histopathology of the decorticated pleura and wedge resected parenchyma revealed no occurrence of bullae, blebs, or endometrial tissue. Twenty days after that operation her complaints recurred; chest x-ray revealed a right pneumothorax, and she was referred to our clinic.

According to medical history, she had no comorbidities except for a fibroadenoma in her right breast, and infertility. She had a smoking habit of 10 pack-years. When her history was queried for the suspicion of catamenial pneumothorax, we found out that she had been in her menstrual cycle during each of the pneumothoraces. In the physical examination, she had an asthenic body type, breath sounds were decreased in the right hemithorax, and she had right axillary scars due to thoracotomy and chest tube. Her body temperature was $36.9^{\circ} \mathrm{C}$, her heart rate was 74 beats $/ \mathrm{min}$, her blood pressure was $100 / 60 \mathrm{~mm} \mathrm{Hg}$, and her breathing frequency was 18 breaths/min. Laboratory and biochemical results were normal. Computed tomography revealed that the pneumothorax in the right hemithorax had caused the lower lobe to collapse (see Fig. 1).

She underwent lateral thoracotomy for the diagnosis of recurrent pneumothorax. During the thoracotomy, thickened parietal pleura and 5-6 defects in the tendinous part of the diaphragm were observed. The defects were about 1-2 $\mathrm{mm}$ in diameter (Fig. 2). No intrathoracic endometri- 

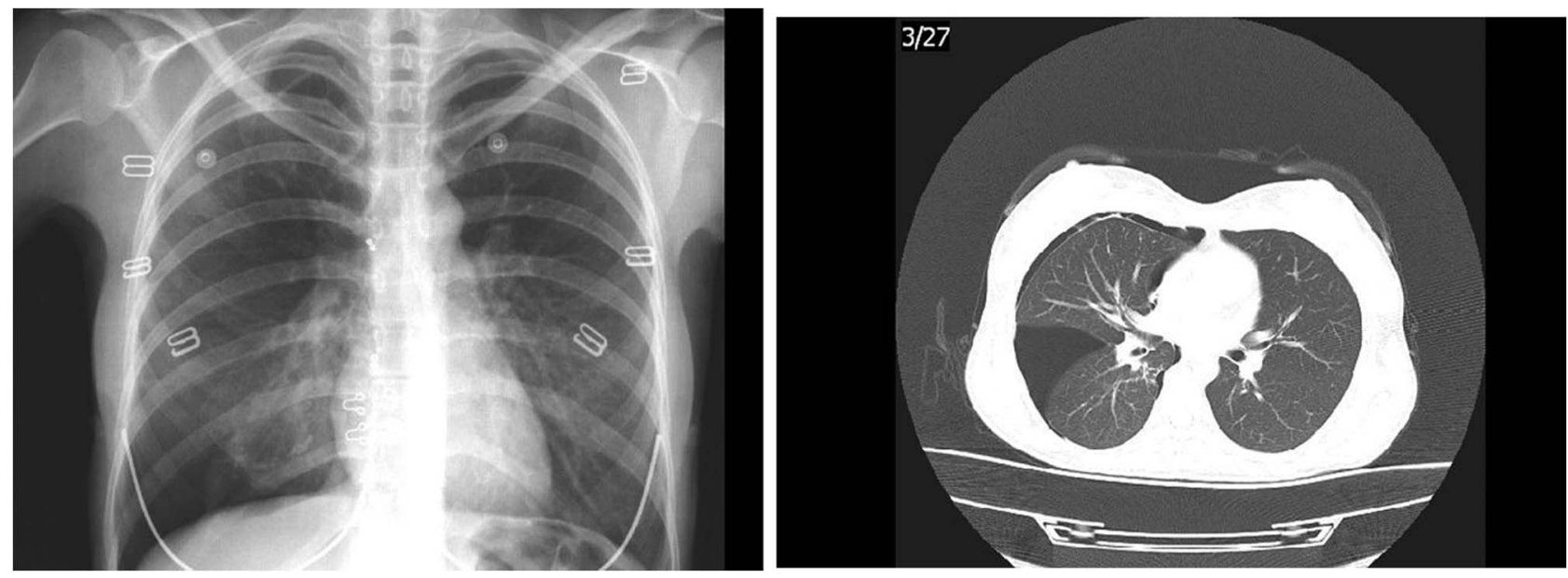

Fig. 1. Chest $\mathrm{x}$-ray and computed tomography reveal pneumothorax in the right hemithorax.

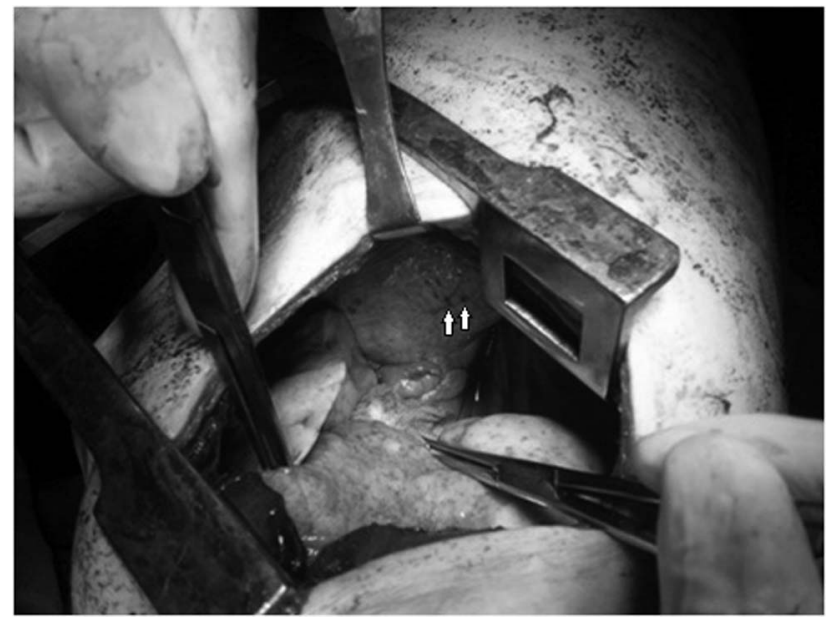

Fig. 2. During thoracotomy, numerous fenestrations were detected on the tendinous part of the right diaphragm (marked with arrows).

osis, blebs, or bullae were visualized. Because of the suspicion of thoracic endometriosis, the diaphragm was partially excised, together with one of the defects (Fig. 3). Parietal pleura was decorticated, and the defects in the diaphragm were repaired with interrupted silk sutures. She was discharged on postoperative day 6. Histopathology found no endometrial tissue in the diaphragm or pleura (Fig. 4). After discharge she was referred to an obstetrics and gynecology clinic for infertility. Pelvic ultrasonography revealed myoma uteri and endometrial polyp. She underwent laparoscopic myomectomy. No pelvic endometriosis was identified during the procedure. She was in the 12th postoperative month, and no problems or recurrent pneumothorax had occurred.

\section{Discussion}

Catamenial pneumothorax is a type of spontaneous pneumothorax, the clinical presentation of which involves recurrent pneumothorax during or preceding menstruation. The presenting symptoms are chest pain, shortness of breath, and (rarely) hemoptysis. More than one pneumothorax episode is usually observed before diagnosis. Catamenial pneumothorax is usually right-sided, and recurrence disposition during menses is high. Symptoms of pelvic endometriosis have been observed in most patients..$^{1,4}$

Endometriosis is defined as the occurrence of endometrial tissue outside the uterus. The disease is usually observed in women of reproductive age, and causes pelvic pain and infertility. Catamenial pneumothorax (73\%), catamenial hemothorax (14\%), catamenial hemoptysis (7\%), and parenchymal lesions $(6 \%)$ are the most common presentation of thoracic endometriosis. ${ }^{4,5}$ Catamenial pneumothorax is categorized as either endometriosis-related or non-endometriosis-related. Alifano et al reported that the incidence of non-endometriosis-related catamenial pneumothorax, which developed due to the diaphragmatic defects, was 3.5\% (4/114). ${ }^{5}$ Our patient was in that group.

Although the exact pathophysiology of catamenial pneumothorax is still unknown, there are 3 main theories. According to the microembolization theory, endometrial cells reach the pleural cavity through the venous system or the transdiaphragmatic lymphatics. Most of the pleural lesions are right-sided, and cyclical necrosis of the pulmonary foci may cause air leaks. In the physiologic hypothesis the suggestion is that the high levels of prostaglandin F2 during ovulation causes vasoconstriction and ischemia in the lung tissue, then bronchospasm causes alveolar rupture and pneumothorax. In the transgenital-transdiaphragmatic passage of air theory, it is suggested that diaphragmatic fenestrations, which have developed related or unrelated to the endometrial tissue, allow air to pass from the abdominal cavity into the pleural cavity, in the absence of a cervical mucus plug. ${ }^{4,6}$ This latter theory explains the 


\section{Recurrent Catamenial Pneumothorax Caused by Diaphragmatic Fenestration}

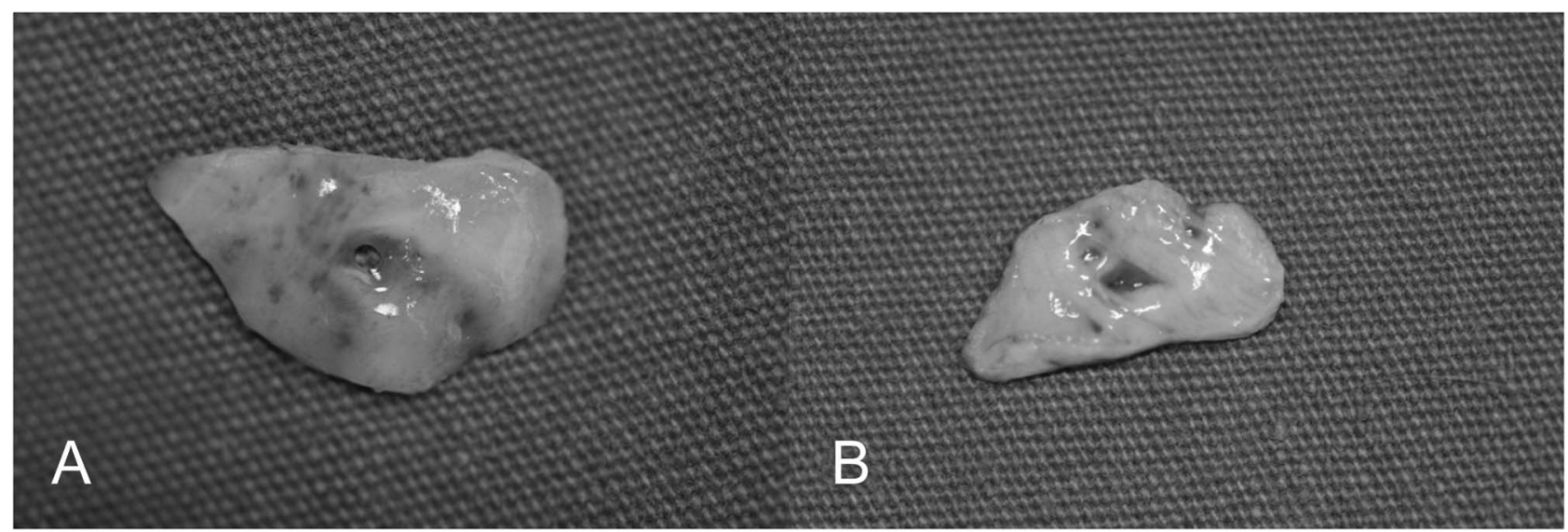

Fig. 3. Excised diaphragm shows the defect on the pleural surface $(A)$ and the peritoneal surface (B).

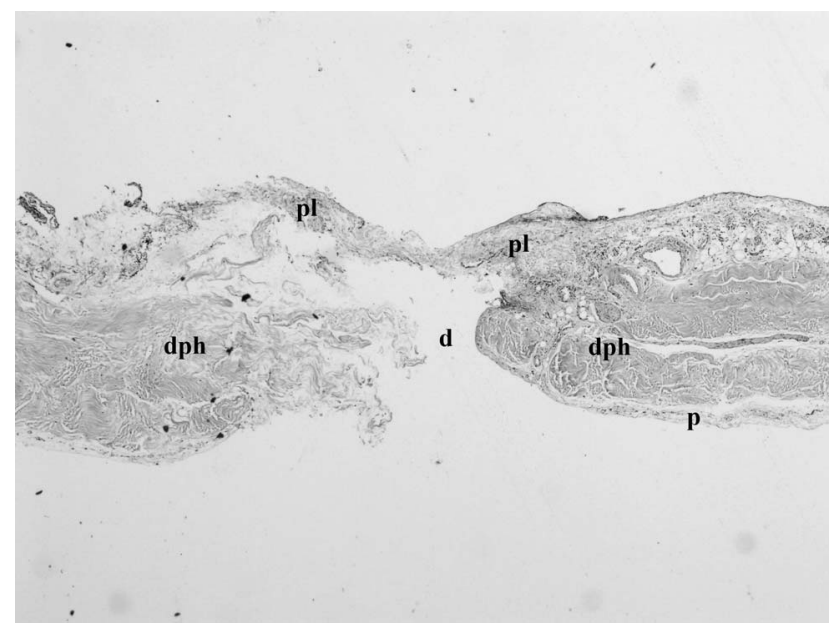

Fig. 4. Diaphragm biopsy shows defect (d), diaphragm (dph), pleura (pl), and peritoneum (p).

non-endometriosis-related catamenial pneumothorax better. However, it fails to explain why pneumothorax does not develop in every menstrual cycle. ${ }^{5}$

The characteristic lesions of catamenial pneumothorax are diaphragmatic defects and/or nodules on the diaphragm, and/or on the visceral and/or parietal pleura, and even one of these is enough for the diagnosis. Computed tomography and magnetic resonance imaging are frequently employed in the diagnosis of catamenial pneumothorax, but computed tomography is insufficient for the identification at diaphragmatic implants and fenestrations. Magnetic resonance imaging is frequently employed in patients whose medical history is suspicious for catamenial pneumotho$\operatorname{rax} .{ }^{3-6}$

The endometrial implants can be single or multiple, and are usually found on the diaphragm. The diaphragmatic defects can be single or multiple, and are mainly located at the tendinous part of the diaphragm. They are usually identified intraoperatively, and thus transdiaphragmatic passage of air is proven. ${ }^{6-8}$ Alifano et al reported that the rates of pleural endometriosis and diaphragmatic defects in patients with catamenial pneumothorax had been quite low in the previous publications, and they attributed this to the treatment methods employed in the past and to the widespread use of thoracoscopy in recent years. ${ }^{5}$

In cases of suspected catamenial pneumothorax, visceral and parietal pleura should be examined for endometrial implants, bullae, and blebs, and the diaphragm should be examined for defects. Among women who underwent routine surgical treatment for recurrent pneumothorax, the rate of catamenial pneumothorax diagnosis reaches $25 \%$. The rate of apical bullae and blebs in patients with catamenial pneumothorax is low $(30 \%))^{4,5}$

Treatment options for catamenial pneumothorax include tube thoracostomy in every episode, suppression of ovulation through oral contraceptives or low-dose androgens, chemical pleurodesis, hysterectomy and bilateral oophorectomy, and pleural abrasion or pleurectomy. Today, video-assisted thoracoscopic surgery has an important role in the diagnosis and treatment of catamenial pneumothorax, but surgical techniques differ, and include resection of the diaphragm or plication of the fenestrations identified during thoracoscopy, placement of mesh over the fenestrations, repair with surgical adhesive (eg, BioGlue), removal of the endometrial foci, and pleurodesis. ${ }^{3-6,9}$

The postoperative recurrence rate is $30-40 \%$. To prevent recurrence, diaphragmatic defects should certainly be closed, when necessary the diaphragm should be excised, and pleurodesis or pleurectomy should be included. The combination of surgical and hormonal treatment decreases the recurrence rate. ${ }^{4-6}$ In their series, Ciriaco et al identified recurrence in 4 patients out of 10. As in our patient, 2 of those patients had undergone operations in other medical centers, and diaphragmatic defects were identified during a later operation. ${ }^{10}$ 


\section{Recurrent Catamenial Pneumothorax Caused by Diaphragmatic Fenestration}

\section{Teaching Points}

- Catamenial pneumothorax is observed in women of reproductive age and recurs just before or during a menstrual period. Catamenial pneumothorax should be considered in a young female presenting with recurrent spontaneous pneumothorax.

- Catamenial pneumothorax is classified as either endometriosis-related or non-endometriosis-related.

- Magnetic resonance imaging is superior to computed tomography for diagnosing catamenial pneumothorax.

- Women presenting with recurrent pneumothorax should undergo surgical exploration. In patients with suspected catamenial pneumothorax it is important to thoroughly examine the chest cavity and lung parenchyma for endometrial implants and diaphragmatic fenestrations.

- Characteristic surgical findings of catamenial pneumothorax are diaphragmatic defects and/or nodules on the diaphragm and/or on the visceral and/or parietal pleura, and even one of these is enough for the diagnosis. Endometrial implants should be resected and diaphragmatic fenestrations repaired.

- Depending on the patient, hormonal treatment can be supplementary after surgery in patients whose pathology findings confirm endometrial tissue. Hormonal treatment is not sufficient for patients with diaphragmatic fenestration.

\section{ACKNOWLEDGMENTS}

We thank Salih Yilmaz for editing the English in an early version of the manuscript.

\section{REFERENCES}

1. Cowl CT, Dunn WF, Deschamps C. Visualization of diaphragmatic fenestration associated with catamenial pneumothorax. Ann Thorac Surg 1999;68(4):1413-1414.

2. Maurer ER, Schaal JA, Mendez FL Jr. Chronic recurring spontaneous pneumothorax due to endometriosis of the diaphragm. J Am Med Assoc 1958;168(15):2013-2014.

3. Blanco S, Hernando F, Gomez A, Gonzalez MJ, Torres AJ, Balibrea JL. Catamenial pneumothorax caused by diaphragmatic endometriosis. J Thorac Cardiovasc Surg 1998;116(1):179-180.

4. Alifano M. Catamenial penumothorax. Curr Opin Pulm Med 2010; 16(4):381-386.

5. Alifano M, Jablonski C, Kadiri H, Falcoz P, Gompel A, CamilleriBroet S, Regnard JF. Catamenial and noncatamenial, endometriosisrelated or nonendometriosis-related pneumothorax referred for surgery. Am J Respir Crit Care Med 2007;176(10):1048-1053.

6. Visouli AN, Darwiche K, Mpakas A, Zarogoulidis P, Papagiannis A, Tsakiridis $\mathrm{K}$ et al. Catamenial pneumothorax: a rare entity? Report of 5 cases and review of the literature. J Thorac Dis 2012;4(Suppl 1):17-31.

7. Sánchez-Lorente D, Gómez-Caro A, Garcia Reina S, Maria Gimferrer J. Treatment of catamenial pneumothorax with diaphragmatic defects. Arch Bronconeumol 2009;45(8):414-415.

8. Rombolá CA, del Pozo P, Honquero Martínez A, León Atance P. Multiple diaphragmatic fenestration as the only thoracoscopic finding in recurrent pneumothorax. Arch Bronconeumol 2008;44(11): 646. Article in Spanish.

9. Attaran S, Bille A, Karenovics W, Lang-Lazdunski L. Videothoracoscopic repair of diaphragm and pleurectomy/abrasion in patients with catamenial pneumothorax: a 9-year experience. Chest 2013; 143(4):1066-1069.

10. Ciriaco P, Negri G, Libretti L, Carretta A, Melloni G, Casiraghi M et al. Surgical tratment of catamenial pneumothorax: a single centre experience. Interact Cardiovasc Thorac Surg 2009;8(3):349-352. 